\title{
Channel current fluctuations conclusively explain neuronal encoding of internal potential into spike trains
}

\author{
Martin N. P. Nilsson (1) \\ RISE Research Institutes of Sweden, POB 1263, SE-164 29 Kista, Sweden \\ Henrik Jörntell $\oplus^{\dagger}$ \\ Department of Experimental Medical Science, Lund University, SE-221 00 Lund, Sweden
}

(Received 17 December 2020; accepted 15 January 2021; published 17 February 2021; corrected 1 March 2021)

\begin{abstract}
Hodgkin and Huxley's seminal neuron model describes the propagation of voltage spikes in axons, but it cannot explain certain full-neuron features crucial for understanding the neural code. We consider channel current fluctuations in a trisection of the Hodgkin-Huxley model, allowing an analytic-mechanistic explanation of these features and yielding consistently excellent matches with in vivo recordings of cerebellar Purkinje neurons, which we use as model systems. This shows that the neuronal encoding is described conclusively by a soft-thresholding function having just three parameters.
\end{abstract}

DOI: 10.1103/PhysRevE.103.022407

\section{INTRODUCTION}

Hodgkin and Huxley developed their neuron model to describe the propagation of voltage spikes (action potentials) in giant squid axons [1]. This model describes the spike initiation and shape remarkably well, but it is now generally thought that information is conveyed by the intervals between spikes rather than the shape of the spikes. Unfortunately, the model cannot explain the apparent stochastic variability of these intervals in biological neurons in vivo. The importance of spike intervals has been emphasized by experiments using electrical activation of the synaptic input of dendrites, showing that the mean axonal output spike frequency tends to be linear in input [2], down to a limit. Such a function performs soft thresholding $[\max (0, a(x-b))$ for input $x ; a$, $b$ constants] and is known as a rectified linear unit (ReLU). It has recently attracted wide attention as a powerful information processing element in the machine learning and statistics communities [3].

The Hodgkin-Huxley model is an equivalent circuit of ion channels exporting potassium $\left(\mathrm{K}^{+}\right)$and importing sodium $\left(\mathrm{Na}^{+}\right)$. The $\mathrm{K}$ and $\mathrm{Na}$ channels are assumed to be uniformly distributed, which is representative of the axon. However, for the full neuron, the spatial distribution of these channels is nonuniform. The density of functional $\mathrm{Na}$ channels is higher in the axon initial segment than in the cell body (soma) and its dendritic neighborhood [4], and the opposite density holds for the $\mathrm{K}$ channels. To reflect these differences in distribution, we divide the neuron into into a distal compartment covering

\footnotetext{
*Corresponding author: neuronmodel@drnil.com

†henrik.jorntell@med.lu.se
}

the dendrites distal from the soma, a proximal compartment covering the soma and proximal dendrites, and an axon initial segment compartment, as shown in Fig. 1. This corresponds to dividing the Hodgkin-Huxley circuit into three subcircuits by two axial resistors, $R_{d p}$ and $R_{p a}$ [Fig. 2(a)].

\section{RESULTS}

First, we were able to derive the probability density of spike intervals analytically using recent progress in firstpassage time theory. Second, by comparing the theoretical density with in vivo electrophysiological spike train recordings from cerebellar Purkinje neurons, we found excellent matches [e.g., Fig. 2(b)], allowing us to infer not only what the neuron can do, but also what it cannot do. Third, from this density we derived an expression for the encoding (activation) function, forming a soft-thresholding function. Its input, gain (slope), and offset (bias) directly correspond to the three model parameters.

\section{RELATED WORK}

In 1964, Gerstein and Mandelbrot [5] formulated the spike interval distribution as a first-passage time problem, where
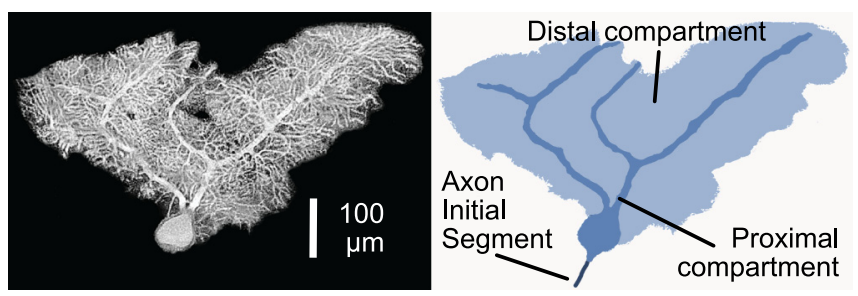

FIG. 1. Confocal photomicrograph of Purkinje neuron. The photo has been edited by removing neighboring cells and enhancing resolution, contrast, and sharpness. The stylized image shows the division into three compartments. 


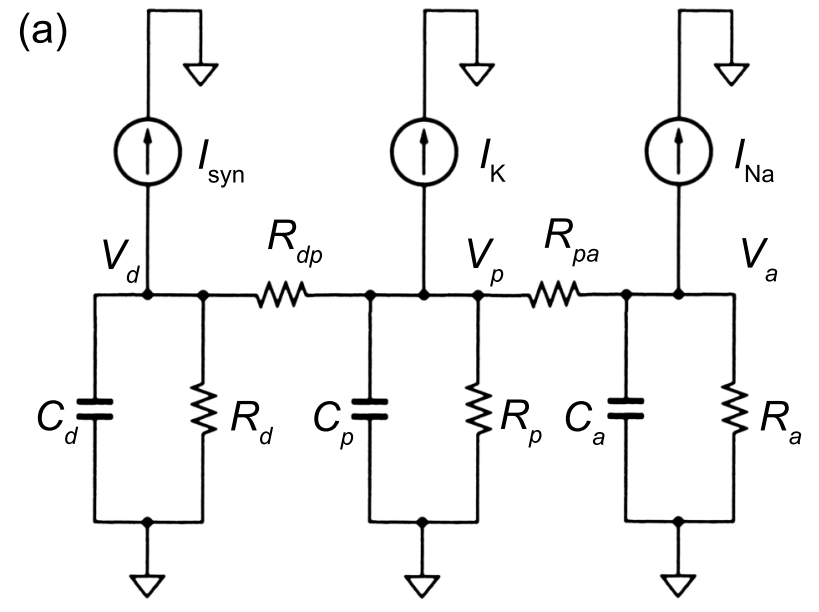

(b)

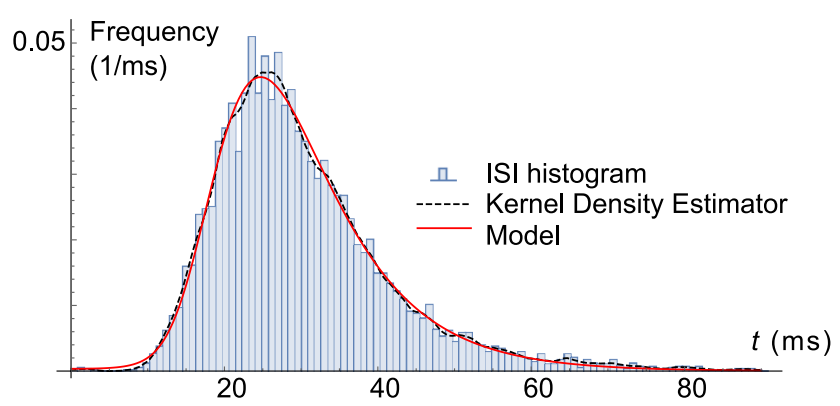

FIG. 2. Circuit model and validation. (a) Division of the Hodgkin-Huxley model into three compartments by resistors $R_{d p}$ and $R_{p a}$. Symbols $R, C, V, I$ denote resistances, capacitances, potentials, and currents, respectively; indexes $d, p, a$ denote distal, proximal, and axon initial segment compartments, respectively. (b) Comparison of the model's first-passage time distribution and inter-spike interval (ISI) histogram of the electrophysiological recording showing a typical, nearly perfect match (recording ID: HWP12).

a spike is generated when the internal potential—described as a stochastic process - crosses a threshold. They used random walks to a fixed boundary, and in 1967, Gluss [6] introduced an exponentially shrinking boundary. However, this early work was hindered by the lack of knowledge of ion channel properties, which was only obtained after the invention of the patch-clamp recording technique in 1976 [7].

From a theoretical point of view, efficient techniques for solving first-passage problems with moving boundaries were not generally known and still require nontrivial mathematical tools today. Consequently, much of the work has focused on empirical (phenomenological) models, which have the inherent advantage of fitting data very precisely. A disadvantage of this approach, compared to mechanistic models, which are based exclusively on biophysical principles, is that only the latter provide explanations, as discussed by Craver [8]. However, it is harder for mechanistic models to fit experimental data [9]. The ultimate challenge-and our objective-is, therefore, to find a conclusive model, i.e., a mechanistic model with the minimal number of parameters that fits data as well as any empirical model.
Two-compartment models have been proposed for biological reasons [10,11], e.g., based on the structural observation that only the distal dendrites have spiny branchlets, and because of the limitations of single-compartment models. Rospars and Lánský [12] noted that the single-compartment integrate-and-fire model counterintuitively resets the memory of the entire neuron for every spike. Naundorf et al. [13] pointed out that biological neurons display a sharp kink at the spike onset, which single-compartment models cannot achieve, but Brette [14] showed that compartmentalization can solve this problem. On the other hand, the difficulty to analyze many-compartment models theoretically has also contributed to the popularity of two-compartment models $[12,15-17]$. Nevertheless, the mathematical treatment has remained difficult [18, includes a review]. A comprehensive review of currently popular models can be found in other work [19].

It is often assumed that the variability is caused by spike arrivals at synapses, but synaptic inputs alone cannot explain spike train variability, because Purkinje neurons fire spontaneously with variable intervals even if synaptic inputs are pharmacologically disabled [20]. It has alternatively been suggested that channel current fluctuations contribute to variability [21,22], and modulated current injection experiments in vitro for cortical interneurons have pointed to an important role of $\mathrm{K}$ channels [23].

\section{THEORETICAL MODEL}

We are only interested in the spike intervals; therefore, we consider only the internal potential between the spikes, in the vicinity of the "resting" potential [25], which we define as zero for simplicity. Standard circuit theory describes the threecompartment model in Fig. 2(a) by the equations

$$
\begin{aligned}
C_{d} \frac{d V_{d}}{d t} & =-\frac{V_{d}}{R_{d}}-\frac{V_{d}-V_{p}}{R_{d p}}-I_{\mathrm{syn}}, \\
C_{p} \frac{d V_{p}}{d t} & =-\frac{V_{p}}{R_{p}}+\frac{V_{d}-V_{p}}{R_{d p}}-I_{\mathrm{K}}-\frac{V_{p}-V_{a}}{R_{p a}}, \\
C_{a} \frac{d V_{a}}{d t} & =-\frac{V_{a}}{R_{a}}+\frac{V_{p}-V_{a}}{R_{p a}}-I_{\mathrm{Na}} .
\end{aligned}
$$

These equations look deceptively linear, but nonlinearities lurk in the channel currents $I_{\mathrm{K}}$ and $I_{\mathrm{Na}}$. $I_{\mathrm{syn}}$ is the synaptic current, $I_{\mathrm{K}}$ is the positive (polarizing) current exported by $\mathrm{K}$ channels in the proximal compartment, and $I_{\mathrm{Na}}$ is the negative (depolarizing) current exported by $\mathrm{Na}$ channels in the axon initial segment. These $\mathrm{Na}$ channels are voltage dependent, $I_{\mathrm{Na}}=I_{\mathrm{Na}_{0}} e^{\alpha V_{a}}$, where $I_{\mathrm{Na}_{0}}$ and $\alpha$ are constants (the latter known as the logarithmic voltage sensitivity) [26].

The potassium current is of premium interest here. It can be divided into two components, $I_{\mathrm{K}}=I_{\text {fast }}+I_{\text {slow }}[20,26-$ 28]. Component $I_{\text {fast }}$ is a strong transient current generated by voltage-gated $\mathrm{K}$ channels and big-conductance calciumgated $\mathrm{K}$ channels, together creating the quickly falling edge of the most recent spike, seen to the left in Fig. 3, which shows a sample simulation of the system. This current decays exponentially and can be written $I_{\mathrm{fast}}=I_{\mathrm{K}_{0}} e^{-t / \tau_{\mathrm{K}}}$, where $I_{\mathrm{K}_{0}}$ is a constant describing the initial current and $\tau_{\mathrm{K}}$ is the time constant. In mice, its mean \pm standard deviation has been 


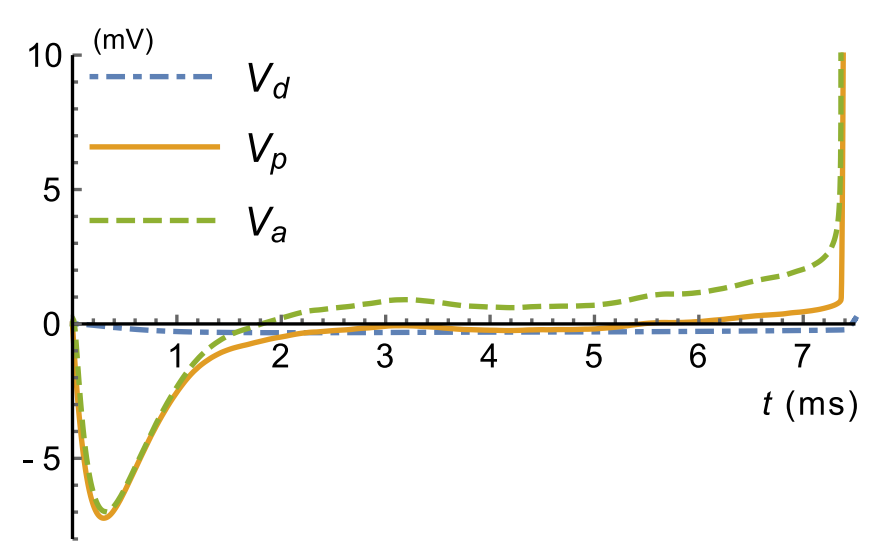

FIG. 3. Simulation example. Solution of model equations (1)(3). Here, a spike is triggered after $7 \mathrm{~ms}$, upon the first threshold passage of $V_{p}$. The generating program is provided in the Supplemental Material [24, section 5].

measured to $0.28 \pm 0.01 \mathrm{~ms}$ (six samples) [20]. The other component, $I_{\text {slow }}$, is weaker but persistent and is generated by the intermediate- and small-conductance calcium-gated $\mathrm{K}$ channels known as SK channels [26,27]. We model this current by the number $n$ of open channels, each passing the constant elementary channel current $i, I_{\text {slow }}=i n$.

Importantly, the SK channels open and close stochastically; therefore, the number of open channels varies over time, $n=n(t)$, creating channel noise. For example, Hirschberg et al. [28] studied clones of the SK-channel subtype found in Purkinje neurons [29] and measured open durations exponentially distributed with mean durations between $1.2 \mathrm{~ms}$ and $11 \mathrm{~ms}$.

Assuming that the ion channels are independent, $n$ can be modeled as a queueing process of Poisson arrivals having rate $\lambda$, representing the channel opening events, and exponentially distributed service duration with mean $\tau$, representing the channel open duration. The equilibrium value $\rho$ of $n$ equals $\lambda \tau$, and we can define a noise current $I_{\mathrm{ch}} \triangleq I_{\text {slow }}-i \rho$ $=i(n-\rho)$.

It has been shown [30] that $(n-\rho) / \sqrt{\rho}$ approaches an Ornstein-Uhlenbeck process for large $\rho$, such that $I_{\mathrm{ch}}$ is described by the stochastic differential equation

$$
\frac{d I_{\mathrm{ch}}}{d t}=-\frac{I_{\mathrm{ch}}}{\tau}+i \sqrt{2 \lambda \tau} \xi
$$

where $\xi$ is zero-mean unit-variance white Gaussian noise.

Assuming stationary synaptic input during a spike interval, we consider $V_{d}$ to be nearly constant, because $\tau_{d}$ is large compared to $\tau_{p}$ and the interval length, where $\tau_{d}$ and $\tau_{p}=C_{p} R_{p} R_{d p} /\left(R_{p}+R_{d p}\right)$ are the time constants for the distal and proximal compartments, respectively. This is supported by our patch-clamp measurements, which show that synaptic input is heavily low-pass filtered on the way to the proximal compartment (Fig. 4). On the other hand, $C_{a} \ll C_{p}$, because the axon initial segment is much smaller than soma, so we model the axon initial segment as a level detector [14].

For subthreshold $V_{p}$, Eq. (2) can be written

$$
C_{p} \frac{d V_{p}}{d t}=\frac{V_{d}}{R_{d p}}-\frac{V_{p}\left(R_{p}+R_{d p}\right)}{R_{p} R_{d p}}-I_{\mathrm{K}},
$$
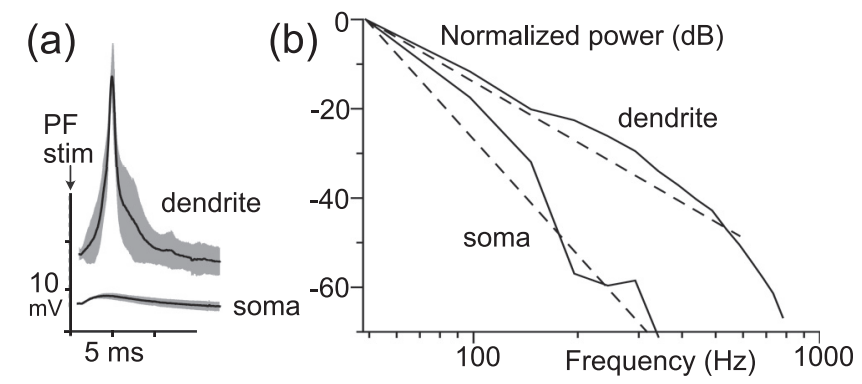

FIG. 4. Measurements. PF = parallel fiber. (a) Mean and standard deviation (in gray) of PF-evoked responses in dendrites and soma (12 samples) of Purkinje neurons when PFs are electrically stimulated. Curves offset vertically for clarity. (b) Dendritic attenuation. Power spectrum of PF-evoked signals in the dendrite and soma. Dashed lines indicate linear regressions.

Equation (5) is a second stochastic differential equation driven by the colored noise $I_{\mathrm{ch}}$ via $I_{\mathrm{K}}$. We can use the fact that $\tau \gg \tau_{p}$ (Llano et al. [11] measured $\tau_{p}$ in the range of $0.2-0.4 \mathrm{~ms}$ in vitro in rat Purkinje neurons), and apply a technique from Stratonovič [31] using stochastic equivalence to transform $V_{p}$ to an Ornstein-Uhlenbeck process $x$. This conversion is described in detail in the Supplemental Material [24, section $2]$, and results in the first-passage boundary condition

$$
x(t)<\sigma_{\infty}+\sigma_{1} e^{-t / \tau}+\sigma_{2} e^{-t / \tau_{\mathrm{K}}}+\sigma_{3} e^{-t / \tau_{p}}
$$

for $x$ defined by

$$
\frac{d x}{d t}=-\frac{x}{\tau}+\sqrt{2} \xi
$$

where $\sigma_{\infty}, \sigma_{1}, \sigma_{2}$, and $\sigma_{3}$ are dimensionless constants. $\sigma_{\infty}$ is the normalized distance between the spiking threshold and the resting potential, and becomes a linear function of the input.

The Na channels are inactivated for some time after a spike. During this refractory period, the probability of spiking is low, hiding the last two exponential terms of Eq. (6). We can include the refractory period in the model by capping the boundary with the ceiling $\sigma_{\max }$ by using a smoothed minimum or log-sum-exp function,

$$
\sigma(t) \triangleq-\log \left[e^{-\sigma_{\max }}+e^{-\sigma_{\exp }(t)}\right]
$$

where, letting $\sigma_{0} \triangleq \sigma_{\infty}+\sigma_{1}$,

$$
\sigma_{\exp }(t) \triangleq \sigma_{\infty}+\left(\sigma_{0}-\sigma_{\infty}\right) e^{-t / \tau} .
$$

We can now write the boundary condition as

$$
x(t)<\sigma(t)
$$

having the free parameters $\tau, \sigma_{\infty}, \sigma_{\max }$, and $\sigma_{0}$. Given a recording, the identification of these is an inverse problem, requiring a fast method for solving forward first-passage problems with moving boundaries. Such a method based on time-variable eigenfunction expansion was recently proposed [32], and details are provided in the Supplemental Material [24, section 2].

The solution of the first-passage time problem is the theoretical spike-interval cumulative distribution function $F(t)$. We can calculate the parameter values by fitting the probability density $d F / d t$ to the inter-spike interval 
histogram-or better, the kernel density estimator-of a recorded spike train.

\section{EXPERIMENTAL VALIDATION}

We performed two series of recordings: the first to verify that synaptic input is low-pass filtered on the way to the soma and the second to collect spike trains. We carried out the recordings as previously described [33-35], but here for Purkinje neuron dendrites and somata in lobule $\mathrm{V}$ of the pars intermedia of the cerebellar anterior lobe of cats. All procedures on animals were approved in advance by the Malmö/Lund Animal Research Ethics Committee (permit number and approval-ID: M32-09).

To investigate dendritic damping in vivo we used local electrical stimulation of parallel fibers by single $0.2-\mathrm{ms}$ square current pulses and whole-cell recordings from dendrites and somata [12 samples each; Fig. 4(a)]. We included only recordings containing low-threshold responses to avoid triggering spikes that would bias the measurements. Somatic recordings_-performed with lowered internal potential ( $h y$ perpolarization), again to avoid action potentials-disclosed slow, flat responses to the stimulus [Fig. 4(a)]. The power spectra of the signals recorded from dendrites and soma are shown in Fig. 4(b), indicating a roll-off of $20 \mathrm{~dB}$ per decade and confirming that the effect of distal synaptic input on high frequency variability at the soma is small.

To collect spike trains, we used long ( $>1000$ intervals, corresponding to $\approx 23 \mathrm{~s}$ total duration) extracellular recordings of Purkinje neuron somata (14 samples) without stimulation while ensuring their stationary behavior. By severing the cerebro-cerebellar connection, we ascertained that the recordings could be performed undisturbed by internally generated signals from the neocortex or by any degrading influences on the neuronal network or neuron membranes due to anesthetics. Details of the recordings, including the recorded spike trains, can be found in the Supplemental Material [24, sections 3 and 6].

We analyzed the spike train data (14 samples) and estimated parameter values by fitting the theoretical probability density $d F / d t$ to the spike train kernel density estimators. As the fitness function, we used $1-R^{2}$, where $R^{2}$ is the coefficient of determination. Details of the statistical procedures and the source code of the analysis tool INSTANT are given in the Supplemental Material [24, sections 4 and 5].

As a result, we found that the fits were excellent, achieving an average $R^{2}$ of 0.993 , where $R^{2}=1$ means identity. An example is illustrated in Fig. 2(b), and this match is not exceptional; the fit is similarly good for all measured spike trains, as can be seen in the Supplemental Material [24, section 7]. The optimal value of $\sigma_{\max }$ was nearly the same for all spike trains. As the fitness was also rather insensitive to this value, we fixed $\sigma_{\max }$ to 3.3 as a hyper-parameter, reducing the number of model parameters to three. The mean \pm standard deviation of the time constant $\tau$ was $5.5 \pm 2.7 \mathrm{~ms}$, conforming with the value $8.6 \pm 2.2 \mathrm{~ms}$ (ten samples) we measured intracellularly for another set of Purkinje neurons.

To investigate the linearity of the activation function, we computed the output spike rate (frequency) as the reciprocal

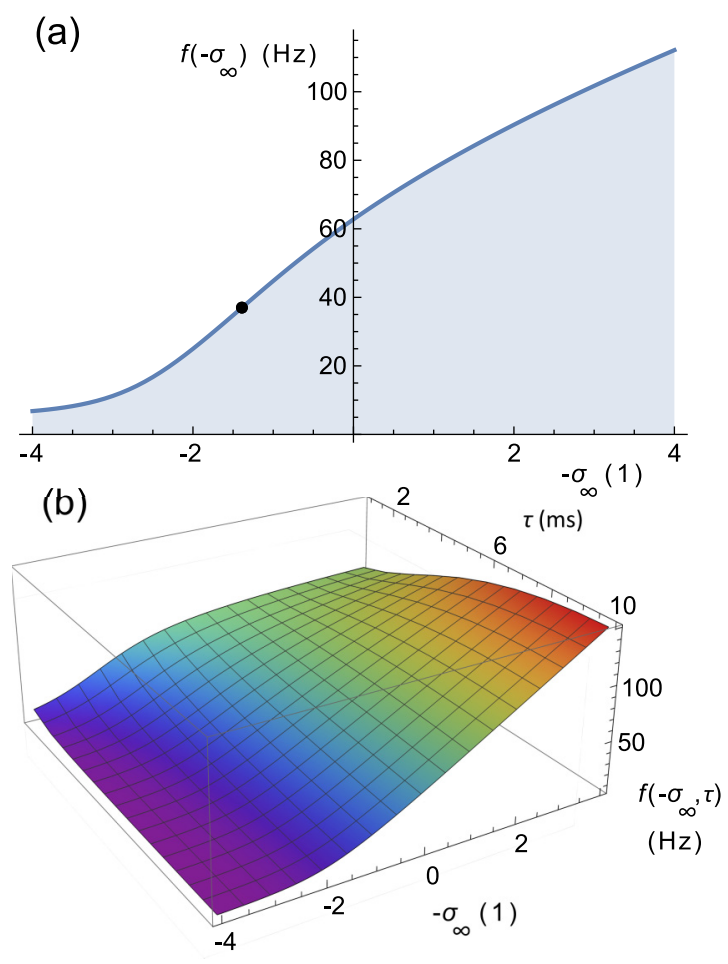

FIG. 5. Linearity. (a) Activation function. Output frequency as a function of input current $\left(I_{\text {syn }}\right.$, effectively $\left.-\sigma_{\infty}\right)$. The black dot identifies recording NWP13. (b) Gain control. The slope is controlled by the open duration $\tau$.

of the mean spike interval duration,

$$
f=\frac{1}{E[t]}=\left\{\int_{0}^{\infty}[1-F(t)] d t\right\}^{-1}
$$

This expression yields a function of $-\sigma_{\infty}$, which is essentially a rectified linear unit with a rounded corner [Fig. 5(a)] for measured biological values of the parameters. This property holds for all measured neurons and is in agreement with previous experimental results [2]. The parameter $\tau$ influences the slope of $f$ but not its linearity as such for measured biological values of $\tau$ [Fig. 5(b)].

\section{DISCUSSION}

The significant congruence between the theoretical and experimental spike distributions means that the receiving neuron cannot reliably determine values of additional parameters beyond the triple $\left(\sigma_{\infty}, \tau, \sigma_{0}\right)$, unless the lengths of spike trains are increased beyond a reasonable limit, i.e., the duration of stationarity.

Adding a fourth parameter is thus redundant and would reduce the accuracy of the estimates of the other parameters without any substantial improvement in fitness. Conversely, we cannot further remove a parameter without gravely mismatching the theoretical and experimental distributions. In other words, the Purkinje neuron computes the soft-thresholding activation function and that is it-no more, no less. Whereas the neuronal encoding function appears simple - having only three parameters - its expressional power should not be underestimated. In a large ensemble of 
neurons operating together, such a function can be surprisingly powerful [3].

What would the biological function of spike train variability be for stationary input? The high-frequency jitter in spike timing may at first appear useless, because it will be removed when the spike train is low-pass filtered by the receiving neuron. Nevertheless, such jitter does have the important function of improving the temporal resolution and reducing the distortion of low-intensity signals in pulse frequency modulated (PFM) transmission schemes. Were it not for the jitter, only high-intensity signals, corresponding to short spike intervals, could be transmitted with high resolution. This principle relates to stochastic resonance [36], which can be illustrated in the following way: Suppose that we want to digitize an analog signal by a comparator. Used directly, it delivers only one-bit (spatial) resolution, but if we dither the analog signal by adding some high-frequency zero-mean random noise, and then digitally low-pass filter the comparator's output, we can increase the resolution.

Why the emphasis on stationary inputs? In this work, we have gone to great lengths to guarantee stationarity of neuronal input (e.g., by statistical testing and isolating the cerebellum from cortical input). The major reason for this approach is that we wanted to eliminate the influence of potential error sources, such as mental processes. However, given an accurate model for stationary input, we can directly generalize to the non-stationary case by taking the distal compartment's low-pass action into account. It removes the high-frequency content of input to make it vary only slowly (pseudostationary). While stationary input is represented by a constant threshold distance $\sigma_{\infty}$ via a linear function, nonstationary input is represented by a slowly time-varying threshold distance $\sigma_{\infty}(t)$, still via the same linear function, but now following the low-frequency component of the input. More technical discussions of the close relation between stationary and nonstationary behaviors can be found in the papers by Johnson $[37,38]$.

Although Purkinje neurons offer good model systems, displaying the whole range of cytological features that are found in neurons in general [39], there are certainly other types whose behavior differs greatly from Purkinje neurons. Such neurons need to be studied separately to draw conclusions about their activation functions.

\section{CONCLUSIONS}

The K-channel current fluctuations between spikes, together with a division of the Hodgkin-Huxley model into three compartments, explain both the variation in spike intervals and the rectified linear unit characteristics, which are two features that are essential for the neuron as an information processing element. The three-compartment model allows for analytical derivation of the interspike interval histogram, which matches in vivo recordings well. The distal first compartment integrates the input; the proximal second compartment generates a ramp and probes it stochastically; and the third, axon initial segment third compartment performs threshold detection and spike initiation.

The Purkinje neuron implements a rectified linear unit with a rounded corner, comprising only three essential parameters: the internal potential, accumulating the synaptic input; the proximal K-channel mean open duration, providing the prominent time constant and the gain of the activation function; and a delay parameter, corresponding to the bias of the activation function.

\section{ACKNOWLEDGMENTS}

This research was funded in part by the European Commission Sixth Framework Program project SENSOPAC under Grant Agreement No. 28056 and European Commission Seventh Framework Program project THE under Grant Agreement No. 248587.

M.N. developed the model and analysis, performed simulations, and wrote the spike-train analysis toolbox. H.J. performed the biological experiments and recordings. M.N and H.J. discussed the multidisciplinary topic intensely as physicist and biologist, respectively.

The authors declare that there are no competing financial interests.
[1] A. Hodgkin and A. Huxley, Currents carried by sodium and potassium ions through the membrane of the giant axon of Loligo, J. Physiol. 116, 449 (1952).

[2] J. T. Walter and K. Khodakhah, The linear computational algorithm of cerebellar Purkinje cells, J. Neurosci. 26, 12861 (2006).

[3] X. Glorot, A. Bordes, and J. Bengio, Deep sparse rectifier neural networks, in Proceedings of the 14th International Conference on AI and Statistics (AISTATS), PMLR (Fort Lauderdale, FL, USA, 2011), Vol. 15, pp. 315-323.

[4] C. Y.-M. Huang and M. N. Rasband, Axon initial segments: Structure, function, and disease, Ann. N.Y. Acad. Sci. 1420, 46 (2018).

[5] G. L. Gerstein and B. Mandelbrot, Random walk models for the spike activity of a single neuron, Biophys. J. 4, 41 (1964).
[6] B. Gluss, A model for neuron firing with exponential decay of potential resulting in diffusion equations for probability density, Bull. Math. Biophys. 29, 233 (1967).

[7] Single-Channel Recording, 2nd ed., edited by B. Sakmann and E. Neher (Springer, New York, 1995).

[8] C. F. Craver, When mechanistic models explain, Synthese 153, 355 (2006).

[9] J. Ladenbauer, S. McKenzie, D. F. English, O. Hagens, and S. Ostojic, Inferring and validating mechanistic models of neural microcircuits based on spike-train data, Nat. Commun. 10, 4933 (2019).

[10] C. Sotelo, Purkinje cell ontogeny: Formation and maintenance of spines, Prog. Brain Res. 48, 149 (1978).

[11] I. Llano, A. Marty, C. M. Armstrong, and A. Konnerth, Synaptic- and agonist-induced excitatory currents of Purkinje cells in rat cerebellar slices, J. Physiol. 434, 183 (1991). 
[12] J. P. Rospars and P. Lánský, Stochastic model neuron without resetting of dendritic potential: Application to the olfactory system, Biol. Cybern. 69, 283 (1993).

[13] B. Naundorf, F. Wolf, and M. Volgushev, Unique features of action potential initiation in cortical neurons, Nature 440, 1060 (2006).

[14] R. Brette, Sharpness of spike initiation in neurons explained by compartmentalization, PLoS Comput. Biol. 9, e1003338 (2013).

[15] A. F. Kohn, Dendritic transformation of random synaptic inputs as measured from a neuron's spike train-modelling and simulation, IEEE Trans. Biomed. Eng. 36, 44 (1989).

[16] P. C. Bressloff, Dynamics of a compartmental model integrateand-fire neuron with somatic potential reset, Phys. D 80, 399 (1995).

[17] P. Lánský and R. Rodriguez, Two-compartment stochastic model of a neuron, Phys. D 132, 267 (1999).

[18] J. Touboul and O. Faugeras, A characterization of first hitting time of double integral processes to curved boundaries, Adv. Appl. Probab. 40, 501 (2008).

[19] W. Gerstner, W. M. Kistler, R. Naud, and L. Paninski, Neuronal Dynamics: From Single Neurons to Networks and Models of Cognition (Cambridge University Press, Cambridge, 2014).

[20] I. M. Raman and B. P. Bean, Ionic currents underlying spontaneous action potentials in isolated cerebellar Purkinje neurons, J. Neurosci. 19, 1663 (1999).

[21] C. Chow and J. White, Spontaneous action potentials due to channel fluctuations, Biophys. J. 71, 3013 (1996).

[22] E. Schneidman, B. Freedman, and I. Segev, Ion channel stochasticity may be critical in determining the reliability and precision of spike timing, Neural Comput. 10, 1679 (1998).

[23] K. M. Stiefel, B. Englitz, and T. J. Sejnowski, Origin of intrinsic irregular firing in cortical interneurons, Proc. Nat. Acad. Sci. USA 110, 7886 (2013).

[24] See Supplemental Material at http://link.aps.org/supplemental/ 10.1103/PhysRevE.103.022407 for details on the derivation of equations, recording methods, statistical methods, software, raw data, and processed data. Supplemental Material cites additional references [40-44].

[25] Although "resting potential" is the traditional term, Purkinje neurons in vivo do not rest, but are continuously bombarded by input spikes. A clumsier, but more precise naming would be "limiting potential for stationary input and in the absence of output spikes".

[26] B. Hille, Ion Channels of Excitable Membranes, 3rd ed. (Sinauer Associates, Sunderland, MA, 2001).

[27] P. Sah, $\mathrm{Ca}^{2+}$-activated $\mathrm{K}^{+}$currents in neurones: Types, physiological roles and modulation, Trends Neurosci. 19, 150 (1996).

[28] B. Hirschberg, J. Maylie, J. P. Adelman, and N. V. Marrion, Gating of recombinant small-conductance Ca-activated $\mathrm{K}^{+}$channels by calcium, J. Gen. Physiol. 111, 565 (1998).
[29] E. Hosy, C. Piochon, E. Teuling, L. Rinaldo, and C. Hansel, SK2 channel expression and function in cerebellar Purkinje cells, J. Physiol. 589, 3433 (2011).

[30] D. L. Iglehart, Limiting diffusion approximations for the many server queue and the repairman problem, J. Appl. Prob. 2, 429 (1965).

[31] R. L. Stratonovič, Topics in the Theory of Random Noise. Vol. 1, General Theory of Random Processes. Nonlinear Transformations of Signals and Noise, rev. English ed. (Gordon and Breach, New York, 1963).

[32] M. N. P. Nilsson, The moving-eigenvalue method: Hitting time for Itô processes and moving boundaries, J. Phys. A: Math. Theor. 53, 405201 (2020).

[33] H. Jörntell and C.-F. Ekerot, Receptive field plasticity profoundly alters the cutaneous parallel fiber synaptic input to cerebellar neurons in vivo, J. Neurosci. 23, 9620 (2003).

[34] F. Bengtsson, C.-F. Ekerot, and H. Jörntell, In vivo analysis of inhibitory synaptic inputs and rebounds in deep cerebellar nuclear neurons, PLoS One 6, e18822 (2011).

[35] H. Jörntell and C.-F. Ekerot, Reciprocal bidirectional plasticity of parallel fiber receptive fields in cerebellar Purkinje cells and their afferent interneurons, Neuron 34, 797 (2002).

[36] L. Gammaitoni, Stochastic resonance and the dithering effect in threshold physical systems, Phys. Rev. E 52, 4691 (1995).

[37] D. H. Johnson, The relationship of post-stimulus time and interval histograms to the timing characteristics of spike trains, Biophys. J. 22, 413 (1978).

[38] D. H. Johnson, Point process models of single-neuron discharges, J. Comput. Neurosci. 3, 275 (1996).

[39] S. L. Palay and V. Chan-Palay, Cerebellar Cortex: Cytology and Organization (Springer, Berlin, 1974).

[40] J. C. Eccles, R. Llinás, and K. Sasaki, The excitatory synaptic action of climbing fibres on the Purkinje cells of the cerebellum, J. Physiol. 182, 268 (1966).

[41] T. Hastie, R. Tibshirani, and J. Friedman, The Elements of Statistical Learning: Data Mining, Inference, and Prediction, 2nd ed. (Springer, New York, 2009).

[42] S. M. Kay, Fundamentals of Statistical Signal Processing. Vol. 1, Estimation Theory (Prentice Hall PTR, Upper Saddle River, NJ, 1993).

[43] MATLAB, version 8.1.0.604 (R2013a, 32-bit) (The MathWorks Inc., Natick, Massachusetts, USA, 2013).

[44] Mathematica, version 11.3.0.0 (64-bit) (Wolfram Research, Inc., Champaign, Illinois, 2018).

Correction: An inline equation in the last sentence of the paragraph below Eq. (3) contained a minor error and has been fixed. 